Friedrich von Hardenberg, genannt Novalis 
Herbert Uerlings

\title{
Friedrich von Hardenberg, genannt Novalis
}

\author{
Werk und Forschung
}

J. B. Metzlersche Verlagsbuchhandlung

Stuttgart 
Die Deutsche Bibliothek - CIP-Einheitsaufnahme

Uerlings, Herbert

Friedrich von Hardenberg, genannt Novalis : Werk und Forschung /

Herbert Uerlings. - Stuttgart : Metzler, 1991

ISBN 978-3-476-00779-7

ISBN 978-3-476-03377-2 (eBook)

DOI $10.1007 / 978-3-476-03377-2$

Dieses Werk einschließlich aller seiner Teile ist urheberrechtlich geschützt. Jede Verwertung außerhalb der engen Grenzen des Urheberrechtsgesetzes ist ohne Zustimmung des Verlages unzulässig und strafbar. Das gilt insbesondere für Vervielfältigungen, Übersetzungen, Mikroverfilmungen und die Einspeicherung und Verarbeitung in elektronischen Systemen.

(C) 1991 Springer-Verlag GmbH Deutschland

Ursprünglich erschienen bei J. B. Metzlersche Verlagsbuchhandlung

und Carl Ernst Poeschel Verlag GmbH in Stuttgart 1991 


\section{Inhalt}

NOVALIS, NOVALIS-FORSCHUNG UND

WISSENSCHAFTSGESCHICHTE

NOVALIS-REZEPTION 1800-1945 ……………................................. 15

Novalis-Rezeption bis 1890 .................................................................................. 17

Frühe Wirkungsgeschichte ................................................................................... 17

Novalis in der Literaturgeschichtsschreibung des 19. Jahrhunderts........................ 28

Exkurs: Novalis-Rezeption in England und Amerika .............................................. 75

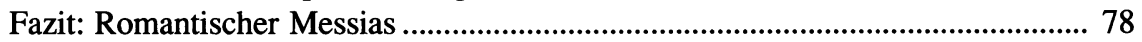

Novalis-Rezeption 1890-1945 ......................................................................... 82

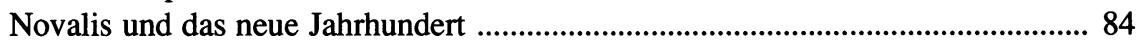

Christliche Novalis-Deutung ............................................................................... 90

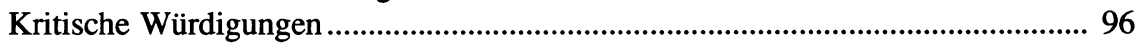

Exkurs: Novalis-Rezeption in Frankreich ............................................................. 100

\section{DAS THEORETISCHE WERK 1: GRUNDLEGUNG DER}

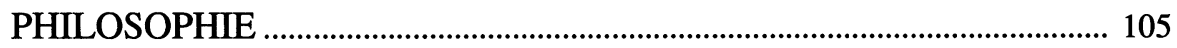

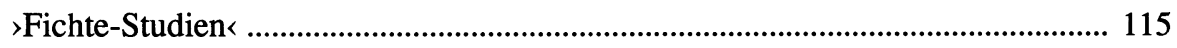

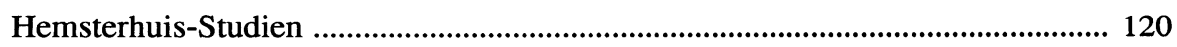

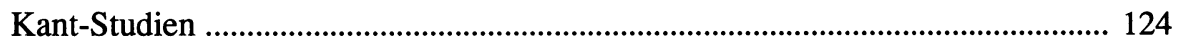

Vorarbeiten zu verschiedenen Fragmentsammlungen .......................................... 126

Das >Allgemeine Brouillon < ............................................................................ 131

$\mathrm{Zu}$ einzelnen Aspekten ................................................................................... 136

Intellektuelle und intellektuale Anschauung ........................................................ 136

Einbildungskraft und Poesie ........................................................................... 140

DAS THEORETISCHE WERK 2: NATURPHILOSOPHIE ..................... 147

Grundlegung ................................................................................................... 149

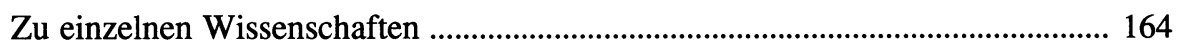

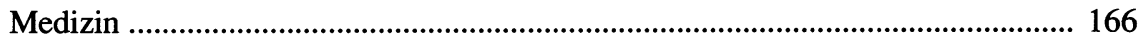

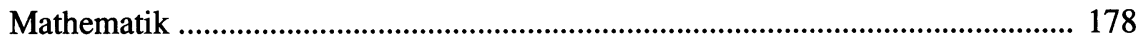

Enzyklopädistik ................................................................................................. 184 
DAS THEORETISCHE WERK 3: ASPEKTE DER ÄSTHETIK 197

Sprachtheorie

Fragment-Ästhetik

ZWISCHENRESÜMEE: Narrative Konstruktion immanenter Transzendenz ... 229

LYRIK

Jugenddichtung und Jugendlyrik (1788-1794)

Tennstedt und Grüningen (1795-1797)

Freiberger Lyrik (1798/99)

>Geistliche Lieder<

Entstehungszusammenhang

$\mathrm{Zu}$ einzelnen Liedern

Exkurs: Frühromantische Religion - Hardenberg und Schleiermacher

>Hymnen an die Nacht

Entstehung und Deutung

Gesamtdeutungen

Zyklus und Struktur

Einzelfragen

Romantiker/Klassiker

Prosagedicht

Mythos, Geschichtsschreibung oder Poesie?

Exkurs: Narrative Konstruktion immanenter Transzendenz in der

5. >Hymne an die Nacht

Späte Lyrik 311 320

Kontext und Modernität

EPIK 351

>Die Lehrlinge zu Sais 353

Zur Einheit des Textes

Das Märchen von Hyazinth und Rosenblüte 360

Neuere Gesamtinterpretationen 366

Theorie des Märchens 383

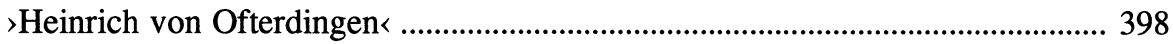

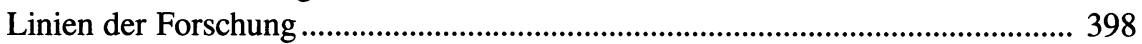

Der Traum von der blauen Blume ................................................................... 406

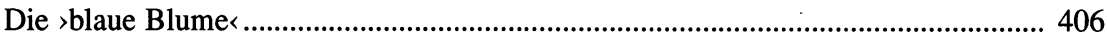

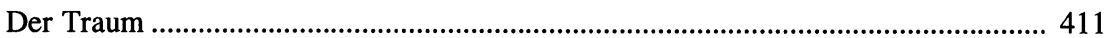

Form und Poetik des Romans ........................................................................... 419 
Zur Form des >Heinrich von Ofterdingen

Dichtungsauffassung im Roman ...................................................................... 431

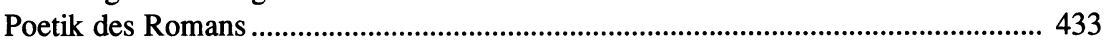

>Wilhelm Meisters Lehrjahre ‘....................................................................... 444

>Meister<-Lektüre und >Meister<-Kritik ................................................................... 444

>Wilhelm Meisters Lehrjahre< und >Heinrich von Ofterdingen< ................................ 449

Der , Ofterdingen < - Ein Bildungsroman? ............................................................. 450

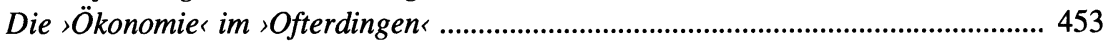

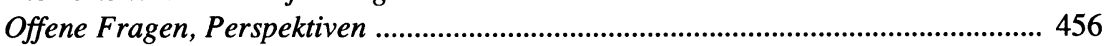

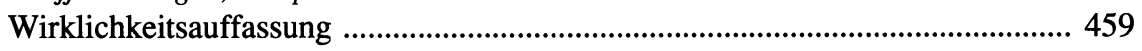

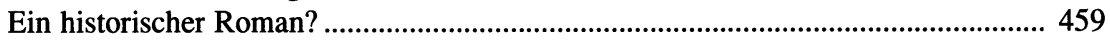

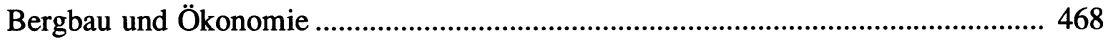

>Indirektes Werkzeug` oder >Zauberstab`? .............................................................. 477

Diskurstheoretische Ansätze ............................................................................ 486

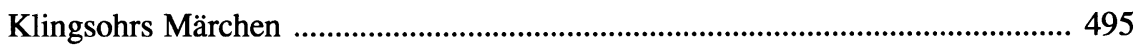

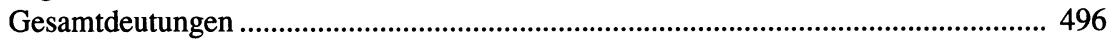

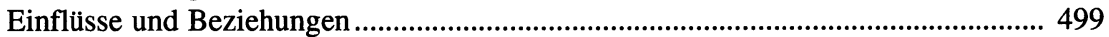

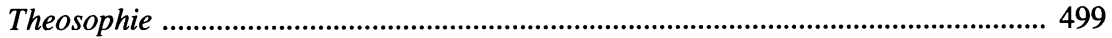

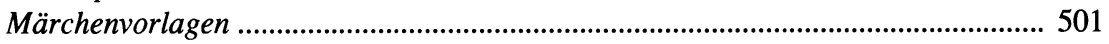

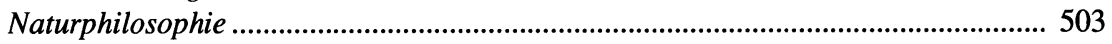

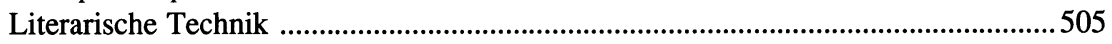

Semiotik und Diskursanalyse ......................................................................... 507

Das Märchen als >Allegorie ‘................................................................................... 507

Zur Psychoanalyse des Märchens ..................................................................... 513

>POLITISCHES`WERK UND GESCHICHTSPHILOSOPHIE .............. 521

>Politische< Rezeption 1890-1945 ………………………….................................. 523

Hardenberg-Forschung in der DDR ............................................................... 542

Politik, Geschichtsphilosophie und Utopie ....................................................... 559

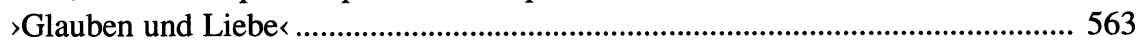

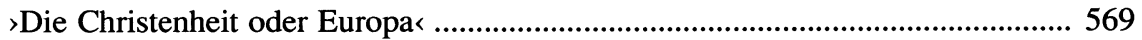

Geschichtsphilosophie und Utopie ............................................................... 579

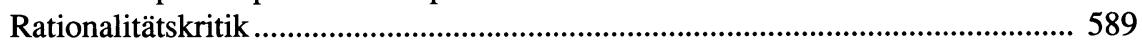

Noch einmal: >Romantik und Konservatismus $<$.............................................. 591

>Politik und Moral oder >Utopiereflexion<? ......................................................... 595

Narrative Konstruktion einer erhofften Tendenz ............................................. 609

EPILOG: Konstruktion oder Dekonstruktion? .................................................615

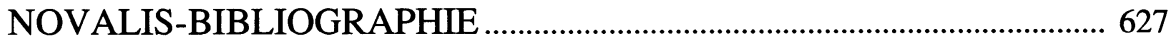




\section{Hinweis zur Zitierweise}

Zitiert wird nach:

Novalis. Schriften. Die Werke Friedrich von Hardenbergs. Begründet von Paul Kluckhohn und Richard Samuel. Herausgegeben von Richard Samuel in Zusammenarbeit mit Hans-Joachim Mähl und Gerhard Schulz. 3., nach den Handschriften ergänzte, erweiterte und verbesserte Auflage in vier Bänden mit einem Begleitband. Stuttgart, Bd. 1: 1977, Bd. 2: 1981, Bd. 3: 1983. Bd. 4 wird nach der 2. Aufl. 1975, Bd. 5 nach der 1. Aufl. 1988 zitiert.

Dabei wurden die Zeichen dieser Ausgabe übernommen:

[ ] von den Herausgebern hinzugefügt

$<>$ von Novalis gestrichen

kursiv von Novalis einmal unterstrichen

ge s p e r $t$ kursiv von Novalis doppelt oder mehrfach unterstrichen

Die Zitatnachweise erfolgen im laufenden Text in runden Klammern nach dem Muster (Bandzahl,Seite:Nr. einer Aufzeichnung).

\section{KFSA}

Friedrich Schlegels Werke werden unter der Sigle KFSA zitiert nach:

Kritische Friedrich-Schlegel-Ausgabe. Hg. v. Ernst Behler unter Mitwirkung von Jean-Jacques Anstett, Jakob Baxa, Ursula Behler, Liselottte Dieckmann, Hans Eichner, Raymond Immerwahr, Robert L. Kahn, Eugène Susini, Bertold Sutter, A. Leslie Willson u.a. Mitarbeitern. Paderborn $1958 \mathrm{ff}$.

Ein Verzeichnis der Zeitschriften-Siglen findet sich auf S. 659. 


\section{Vorurtheile der Gelehrten.}

Den meisten dieser

Caracterzüge liegt gemeiner Egoïsm zum Grunde - und den meisten stehn auch Gegenvorurtheile gegen über.
1. Hang zur Eigenthümlichkeit. (originalitaetssucht.)

Damit steht der Streit um die erste Entdeckung in Verbindung.

2. Pretension auf Consequenz und Infallibilität.

3. $\mathrm{Ha}$ der Autoritaet.

4. Verachtung der Nichtgelehrten.

5. Eifersucht und Verkleinerungssucht der Collegen.

6. Verachtung der andern Wissenschaften.

7. Übertriebne Bewunderung der Mühseligkeit.

8. Sucht, alles alt und schon dagewesen zu finden - und deshalb zu verachten.

9. Verachtung alles dessen, was nicht gelehrt oder gelernt werden kann (Hieher ihr Religions und Wunderhaß - ihr Dichterhaß etc.). 


\section{Danksagung}

Ich danke

Karl Otto Conrady, Köln, der diese Arbeit angeregt und auf vielerlei Weise dafür gesorgt hat, daß sie geschrieben werden konnte,

dem Freunde Norbert Finzsch, Washington, der auch in harten Zeiten in mir die Überzeugung lebendig gehalten hat, daß das ausgehende 18. Jahrhundert als Beginn der historischen Moderne die Zeit eines faszinierenden Epochenumbruchs gewesen ist,

der Eichendorff-Gesellschaft, die mir gleich zweimal Gelegenheit gegeben hat, Ergebnisse dieser Arbeit zur Diskussion zu stellen,

Hans-Joachim Mähl, Kiel, und Gerhard Schulz, Melbourne, für ihre freundliche Unterstützung und

den Freunden und Freundinnen, denen Novalis Hekuba geblieben ist und bleiben wird, ohne die dieses Buch aber auch nicht hätte geschrieben werden können.

Köln, im April 1991 